\title{
The dS swampland conjecture with the electroweak symmetry and QCD chiral symmetry breaking
}

\author{
Kiwoon Choi, Dongjin Chway and Chang Sub Shin \\ Center for Theoretical Physics of the Universe, Institute for Basic Science, \\ Daejeon 34051, South Korea \\ E-mail: kchoi@ibs.re.kr, djchway@gmail.com, csshin@ibs.re.kr
}

Abstract: The dS swampland conjecture $|\nabla V| / V \geq c$, where $c$ is presumed to be a positive constant of order unity, implies that the dark energy density of our Universe can not be a cosmological constant, but mostly the potential energy of an evolving quintessence scalar field. As the dark energy includes the effects of the electroweak symmetry breaking and the QCD chiral symmetry breaking, if the dS swampland conjecture is applicable for the low energy quintessence potential, it can be applied for the Higgs and pion potential also. On the other hand, the Higgs and pion potential has the well-known dS extrema, and applying the dS swampland conjecture to those dS extrema may provide stringent constraints on the viable quintessence, as well as on the conjecture itself. We examine this issue and find that the pion $\mathrm{dS}$ extremum at $\cos \left(\pi_{0} / f_{\pi}\right)=-1$ implies $c \lesssim \mathcal{O}\left(10^{-2}-10^{-5}\right)$ for arbitrary form of the quintessence potential and couplings, where the weaker bound $\left(10^{-2}\right)$ is available only for a specific type of quintessence whose couplings respect the equivalence principle, while the stronger bound $\left(10^{-5}\right)$ applies for generic quintessence violating the equivalence principle. We also discuss the possibility to relax this bound with an additional scalar field, e.g. a light modulus which has a runaway behavior at the pion dS extremum. We argue that such possibility is severely constrained by a variety of observational constraints which do not leave a room to significantly relax the bound. We make a similar analysis for the Higgs dS extremum at $H=0$, which results in a weaker bound on $c$.

KeYwords: Effective Field Theories, Cosmology of Theories beyond the SM

ARXiv EPrint: 1809.01475 


\section{Contents}

1 Introduction 1

2 Quintessence couplings to the standard model 3

3 De Sitter swampland conjecture for the pion and Higgs extrema $\quad 7$

3.1 Pion extremum 7

3.2 Higgs extremum 9

4 Effects of the tadpole or runaway of additional scalar fields 10

5 Conclusion $\quad 16$

\section{Introduction}

Motivated by the difficulty of constructing dS vacuum in string theory, recently the authors of [1] proposed a conjecture that the scalar potential in low energy effective theory which has a UV completion consistent with quantum gravity satisfies

$$
M_{\mathrm{Pl}} \frac{|\nabla V|}{V} \equiv \frac{\sqrt{G^{i j} \partial_{i} V \partial_{j} V}}{V} \geq c
$$

over a certain range of scalar fields which can be of $\mathcal{O}\left(M_{\mathrm{Pl}}\right)$, where $G_{i j}$ is the metric of the scalar field kinetic terms in the Einstein frame, $M_{\mathrm{Pl}} \simeq 2.4 \times 10^{18} \mathrm{GeV}$ is the reduced Planck mass, and $c$ is a positive constant of $\mathcal{O}(1)$. Obviously this conjecture constrains the possible form of (approximate) stationary points or flat directions of the scalar potential with a positive energy density. For instance, once applied for the dark energy density of the present Universe [2], it implies that the dark energy can not be a cosmological constant, but mostly the potential energy of a very light evolving scalar field $\phi$ which is often dubbed quintessence [3-5].

If the dS swampland conjecture is applicable for the low energy quintessence potential, it can be applied for the Higgs and QCD pseudo-scalar meson potential also, since the quintessence potential which is identified as the dark energy density includes the effects of the electroweak symmetry and QCD chiral symmetry breaking. On the other hand, the Higgs and pseudo-scalar meson potential involve the well-known dS extrema, e.g. at $H=0$ or $\cos \left(\pi_{0} / f_{\pi}\right)=-1$, where $H$ is the Higgs doublet and $\pi_{0}$ is the neutral pion field with the periodicity $\pi_{0} \equiv \pi_{0}+2 \pi f_{\pi}$, whose present vacuum values are given by $\langle H\rangle=v=174 \mathrm{GeV}$ and $\left\langle\pi_{0}\right\rangle=0$. The existence of such dS extrema may impose strong constraints on the quintessence which can be compatible with the dS swampland conjecture, 
as well as on the parameter $c$ defining the conjecture. ${ }^{1}$ Indeed, it has been pointed out recently [6] that if the Standard Model (SM) sector is completely decoupled from the quintessence field $\phi$, applying the $\mathrm{dS}$ swampland conjecture to the Higgs extremum results in $c \lesssim V(H=v) / V(H=0) \sim 10^{-55}$, which is smaller than the conjectured value $c=\mathcal{O}(1)$ by many orders of magnitude. Yet, one can avoid this bizarre conclusion by assuming proper (fine-tuned) couplings of $\phi$ to the Higgs sector [6], which may allow $c=\mathcal{O}(1)$ and therefore rescue the conjecture. ${ }^{2}$

Motivated by this observation, in this paper we wish to examine the implications of the pion or Higgs dS extrema for the $\mathrm{dS}$ swampland conjecture, while focusing on the possible (model-independent or model-dependent) bound on the parameter $c$. Here we do not question how much plausible it is to have a viable quintessence in the context of string theory, which is an issue extensively discussed in [29,30] a long time ago, and more recently in [31-33]. Instead, we take the most general quintessence potential and couplings at low energy scales, and examine what would be the maximal value of the parameter $c$ allowed by the observational constraints. We then find that the most stringent bound comes from the pion extremum at $\cos \left(\pi_{0} / f_{\pi}\right)=-1$, yielding

$$
c \leq \operatorname{Max}\left[d_{q}+3 d_{g}, V_{\mathrm{eff}} / f_{\pi}^{2} m_{\pi}^{2} \sim 10^{-43}\right],
$$

where $d_{q}$ and $d_{g}$ are the low energy quintessence couplings to the light quarks and gluons defined in (2.7), $V_{\text {eff }} \sim\left(2 \times 10^{-3} \mathrm{eV}\right)^{4}$ is the quintessence potential energy in the present Universe, and $m_{\pi}$ and $f_{\pi}$ are the pion mass and decay constant, respectively. The observational bounds on the quintessence couplings $d_{q}$ and $d_{g}$ depend on whether they respect or violate the equivalence principle. For a specific type of quintessence whose couplings respect the equivalence principle, e.g. a quintessence which couples to the SM sector only through the trace of the energy momentum tensor, we have $d_{q}=d_{g}$. In such case, the quintessence couplings are constrained mainly by the observational bound on the deviation from the general relativity by the quintessence-mediated force in relativistic limit [34], which results in ${ }^{3}$

$$
c<1.4 \times 10^{-2}
$$

for arbitrary form of the quintessence potential. We call such quintessence a metrical quintessence [30] as such a specific form of couplings which respect the equivalence principle may arise through the mixing with the conformal factor of the spacetime metric. However, for more generic quintessence with $\left(d_{g}-d_{q}\right) /\left(d_{g}+d_{q}\right)=\mathcal{O}(1)$, the quintessence couplings are bounded by the non-observation of the violation of the equivalence principle in nonrelativistic limit [36, 37], yielding a much stronger bound

$$
c<2 \times 10^{-5}
$$

\footnotetext{
${ }^{1}$ The dS swampland conjecture might be modified, for instance as in $[7,8]$ and [9], in such a way that the dS extrema that we are discussing are manifestly compatible with the conjecture.

${ }^{2}$ For recent discussions of various implications of the dS swampland conjecture, see [10-28].

${ }^{3}$ All experimental bounds used in this paper are the $95 \%$ confidence level bounds.
} 
again for arbitrary form of the quintessence potential. The bounds on $c$ from the Higgs extremum is weaker than those from the pion extremum, but yet significantly stronger than the results obtained in [6].

In fact, the above bounds on $c$ are obtained while assuming that other scalar fields in the underlying theory can be integrated out without affecting the low energy dynamics around the pion dS extremum. One can then contemplate the possibility that those bounds are relaxed by an additional scalar field, e.g. a light modulus-like scalar, which has a large tadpole or a runaway behavior when the pion field is at the dS extremum. We examine this possibility also, and find that such a light scalar is severely constrained by a variety of observational constraints which practically close the room to significantly relax the above bounds on $c$.

Our bounds on $c$ from the pion extremum appear to have a significant tension with the dS swampland conjecture (1.1) which assumes $c=\mathcal{O}(1)$. We note that the conjecture (1.1) can be modified or refined, for instance as in $[7,8]$ and [9], in such a way to avoid the bounds from the Higgs and pion extrema. Then our results can be interpreted as providing additional motivation for such refinement of the conjecture.

The organization of this paper is as follows. In the next section, we discuss the possible couplings of quintessence to the SM sector and summarize the relevant observational constraints on the quintessence couplings. In section 3, we apply the dS swampland conjecture for the pion and Higgs extrema, and examine what would be the maximal value of $c$ allowed by the observational constraints in the context of the most general form of the low energy quintessence potential and couplings. In section 4, we examine if an additional scalar which has a sizable tadpole or runaway behavior at the pion dS extremum can relax the bound on $c$ obtained in section 3 . Section 5 is the conclusion.

\section{Quintessence couplings to the standard model}

In this section we briefly discuss the possible couplings of the quintessence scalar field $\phi$ to the SM sector, as well as the observational constraints on the couplings. Without loss of generality, using appropriate field redefinitions, one can always move to the Einstein frame and make the kinetic terms of the SM fermions and the Higgs boson to take the $\phi$-independent canonical form. We are interested in the possible non-derivative couplings of $\phi$ to the SM fields in this field basis, which can be encoded in the $\phi$-dependent SM parameters. Then the lagrangian density at some scale $\mu$ above the weak scale can be written as $^{4}$

$$
\begin{aligned}
\mathcal{L}= & \frac{1}{2} \partial_{\mu} \phi \partial^{\mu} \phi+\left|D_{\mu} H\right|^{2}+\bar{\psi}_{L} i \not D \psi_{L}+\bar{\psi}_{R} i \not D \psi_{R}-\frac{1}{4 g_{a}^{2}(\phi)} F^{a \mu \nu} F_{\mu \nu}^{a} \\
& -\left(y_{\psi}(\phi) H \bar{\psi}_{L} \psi_{R}+\text { h.c. }\right)-\lambda(\phi)|H|^{4}+m_{H}^{2}(\phi)|H|^{2}-V_{b}(\phi),
\end{aligned}
$$

\footnotetext{
${ }^{4}$ For simplicity, here we do not consider the quintessence couplings such as $\theta(\phi) F^{a \mu \nu} \tilde{F}_{\mu \nu}^{a}, \partial_{\mu} \phi H^{\dagger} D^{\mu} H$ and $\partial_{\mu} \phi \bar{\psi} \gamma^{\mu} \psi$ as they do not affect our subsequent discussion. Note that the derivative couplings of $\phi$ to $H$ and $\psi$ can affect the scalar field metric $G_{i j}$ that appears in the dS swampland conjecture, but their effects are suppressed by $v / M_{\mathrm{Pl}} \sim 10^{-16}$ or $f_{\pi} / M_{\mathrm{Pl}} \sim 10^{-19}$ and therefore can be safely ignored.
} 
where $V_{b}$ is the $H$-independent bare potential of $\phi$, and $g_{a}(\phi), \lambda(\phi)$ and $y_{\psi}(\phi)$ are generically $\phi$-dependent gauge, Higgs quartic, and Yukawa couplings, respectively.

From the above lagrangian density, one can calculate the low energy consequences of the model, including the effective potential of $\phi$ at cosmic scales and also the low energy couplings of $\phi$ which are constrained by a variety of laboratory, astrophysical and cosmological observations [34-36, 38]. For instance, the low energy quintessence potential can be obtained by integrating out all SM fields, which would result in

$$
\begin{aligned}
V_{\mathrm{eff}}(\phi) & =V_{b}(\phi)+\left\langle\lambda|H|^{4}-m_{H}^{2}|H|^{2}-\left(y_{\psi} H \bar{\psi}_{L} \psi_{R}+h . c\right)+\frac{1}{4 g_{a}^{2}} F^{a \mu \nu} F_{\mu \nu}^{a}\right\rangle+\ldots \\
& =V_{b}(\phi)-\frac{m_{H}^{4}(\phi)}{4 \lambda(\phi)}-\sum_{q=u, d, s} m_{q}(\phi)\langle\bar{q} q\rangle+\mathcal{O}\left(\Lambda_{\mathrm{QCD}}^{4}\right)+\ldots
\end{aligned}
$$

where $\langle\ldots\rangle$ are the expectation values of the SM fields, $\mathcal{O}\left(\Lambda_{\mathrm{QCD}}^{4}\right)$ denotes the contribution from the gluon condensation including the contributions from the heavy quark thresholds effects, and the ellipsis stands for additional contributions including a variety of additional quantum corrections. If $\phi$ is identified as the quintessence scalar field explaining the dark energy of the present Universe, its low energy potential should satisfy [2, 39]

$$
V_{\text {eff }}(\phi) \sim\left(2 \times 10^{-3} \mathrm{eV}\right)^{4}, \quad M_{\mathrm{Pl}} \frac{V_{\text {eff }}^{\prime}(\phi)}{V_{\text {eff }}(\phi)} \lesssim 0.6
$$

over a field range $\Delta \phi \sim M_{\mathrm{Pl}}$, where the prime denotes the derivative w.r.t. $\phi$, and also

$$
V_{\mathrm{eff}}^{\prime \prime}\left(\phi_{0}\right) \lesssim H_{0}^{2} \sim\left(10^{-33} \mathrm{eV}\right)^{2}
$$

where $\phi_{0}$ and $H_{0}$ are the quintessence field value and the Hubble expansion rate of the present Universe, respectively.

As for the couplings of $\phi$ defined at high energy scale $\mu$, one finds

$$
\mathcal{L}_{\phi}(\mu)=\frac{g_{a}^{\prime}}{2 g_{a}^{3}} \phi F^{a \mu \nu} F_{\mu \nu}^{a}-\left(m_{\psi}\left(\frac{y_{\psi}^{\prime}}{y_{\psi}}+\frac{v^{\prime}}{v}\right) \phi \bar{\psi}_{L} \psi_{R}+h . c\right)-m_{h}^{2}\left(\frac{\lambda^{\prime}}{\lambda}+\frac{2 v^{\prime}}{v}\right) \phi h^{2}+\ldots
$$

where $v$ denotes the $\phi$-dependent Higgs vacuum value given by

$$
v^{2}(\phi)=\frac{m_{H}^{2}(\phi)}{2 \lambda(\phi)},
$$

$h$ is the canonically normalized Higgs boson fluctuation, and again the prime denotes the derivative w.r.t. $\phi$. Here the field $\phi$ corresponds to the fluctuation around $\phi_{0}$, and the ellipsis stands for additional couplings which are not relevant for our subsequent discussion.

As they are even weaker than the gravitational coupling, the quintessence couplings are constrained mostly by the macroscopic observations such as the violation of the equivalence principle in non-relativistic limit, the deviation from the general relativity in relativistic limit, or the variation of the fundamental constants, e.g. the fine structure constant [35]. 
However, those constraints apply for the low energy effective couplings of $\phi$ defined at lower energy scale $\mu_{\text {eff }}$, which may be parametrized as

$$
\begin{aligned}
\mathcal{L}_{\phi}\left(\mu_{\mathrm{eff}}\right) & =d_{\gamma} \frac{\phi}{M_{\mathrm{Pl}}} \frac{1}{4 e^{2}} F^{\mu \nu} F_{\mu \nu}-d_{g} \frac{\phi}{M_{\mathrm{Pl}}}\left(T_{\mu}^{\mu}\right)_{\mathrm{QCD}}-\sum_{q=u, d, s}\left(d_{q}-d_{g}\right) m_{q} \frac{\phi}{M_{\mathrm{Pl}}} \bar{q} q \\
& =d_{\gamma} \frac{\phi}{M_{\mathrm{Pl}}} \frac{1}{4 e^{2}} F^{\mu \nu} F_{\mu \nu}-d_{g} \frac{\phi}{M_{\mathrm{Pl}}} \frac{\beta_{s}}{2 g_{s}} G^{i \mu \nu} G_{\mu \nu}^{i}-\sum_{q=u, d, s}\left(d_{q}+\gamma_{m} d_{g}\right) m_{q} \frac{\phi}{M_{\mathrm{Pl}}} \bar{q},
\end{aligned}
$$

where $\left(T_{\mu}^{\mu}\right)_{\mathrm{QCD}}$ is the trace of the energy momentum tensor for the low energy QCD of the light quark flavors $q=(u, d, s)$, and therefore $\beta_{s}$ and $\gamma_{m}$ are the QCD beta function and the mass anomalous dimension, respectively. Here the gluon fields $G_{\mu \nu}^{i}$ are rescaled to have the standard canonical kinetic term as $-\frac{1}{4} G^{i \mu \nu} G_{\mu \nu}^{i}$. Note that $d_{g}$ and $d_{q}$ are defined to be independent of the renormalization scale $\mu_{\mathrm{eff}}$. Also, they can be defined as

$$
\frac{d_{g}}{M_{\mathrm{Pl}}}=\frac{\Lambda_{\mathrm{QCD}}^{\prime}(\phi)}{\Lambda_{\mathrm{QCD}}(\phi)}, \quad \frac{d_{q}}{M_{\mathrm{Pl}}}=\frac{m_{q}^{\prime}\left(\phi, \Lambda_{\mathrm{QCD}}(\phi)\right)}{m_{q}\left(\phi, \Lambda_{\mathrm{QCD}}(\phi)\right)},
$$

where $\Lambda_{\mathrm{QCD}}(\phi)$ is the $\phi$-dependent physical QCD scale and $m_{q}\left(\phi, \Lambda_{\mathrm{QCD}}(\phi)\right)$ is the $\phi$-dependent light quark mass renormalized at $\Lambda_{\mathrm{QCD}}$.

Although the low energy couplings $d_{g}$ and $d_{q}$ are defined to be independent of $\mu_{\text {eff }}$, perturbative calculation of those couplings in terms of the high energy couplings in (2.5) can be done only for $\mu_{\text {eff }}$ where the perturbation theory applies. For later use, let us briefly discuss the perturbative corrections that $d_{g}$ receives from the high energy couplings in (2.5). At one-loop order, the dominant corrections to $d_{g}$ come from the one-loop thresholds of heavy quarks which couple to $\phi$. There can be also a potentially important two loop correction induced by the $\phi-h-h$ coupling in (2.5) and the top quark Yukawa coupling of the Higgs boson $h$. Putting those radiative corrections with the tree level contribution, we find that the low energy coupling $d_{g}$ at $\mu_{\text {eff }}$ just below the charm quark mass is determined by the high energy couplings in (2.5) as follows:

$$
\frac{d_{g}}{M_{\mathrm{Pl}}} \simeq \frac{\left(16 \pi^{2}\right)}{9} \frac{g_{s}^{\prime}(\mu)}{g_{s}^{3}(\mu)}+\frac{2}{27} \sum_{q=t, b, c}\left(\frac{y_{q}^{\prime}}{y_{q}}+\frac{v^{\prime}}{v}\right)-\frac{y_{t}^{2} f(\tau)}{288 \pi^{2}}\left(\frac{\lambda^{\prime}}{\lambda}+2 \frac{v^{\prime}}{v}\right),
$$

where $\tau \equiv 4 m_{t}^{2} / m_{h}^{2}$. Here the second term in the RHS represents the one-loop threshold of heavy quarks, while the third term corresponds to the two-loop threshold involving the Higgs boson and the top quark. We obtained the analytic form of the function $f(\tau)$ for a generic $\tau$ from full two-loop calculations. For $m_{t}=173 \mathrm{GeV}$ and $m_{h}=125 \mathrm{GeV}$, it yields $f\left(4 m_{t}^{2} / m_{h}^{2}\right)=0.21$.

For generic low energy quintessence couplings, the most stringent constraint comes from the violation of the weak equivalence principle (EPV) by the quintessence-mediated force in non-relativistic limit. For instance, using the results of $[37,40,41]$, we find that non-observation of EPV implies

$$
\left(d_{g}+0.093\left(d_{\tilde{q}}-d_{g}\right)+0.00027 d_{\gamma}\right)\left(3.3\left(d_{\tilde{q}}-d_{g}\right)+1.9 d_{\gamma}\right)<2.7 \times 10^{-11},
$$


where

$$
d_{\tilde{q}}=\frac{m_{u} d_{u}+m_{d} d_{d}}{m_{u}+m_{d}}
$$

If we assume that there is no significant cancellation among the different quintessence couplings, e.g.

$$
\frac{d_{\alpha}-d_{\beta}}{d_{\alpha}+d_{\beta}}=\mathcal{O}(1) \quad(\alpha, \beta=g, q, \gamma)
$$

which would be the case for generic forms of low energy couplings, this implies

$$
d_{g}<3 \times 10^{-6}, \quad d_{\tilde{q}}<10^{-5}, \quad d_{\gamma}<2 \times 10^{-4} .
$$

The quintessence coupling to the photon can be constrained by the observational bound on the time-varying fine structure constant also [35], which would result in

$$
d_{\gamma}<3 \times 10^{-7} \frac{M_{\mathrm{Pl}} H_{0}}{\dot{\phi}},
$$

where $H_{0}$ is the Hubble expansion rate today and $\dot{\phi}=d \phi / d t$.

In fact, there is a specific type of quintessence which automatically satisfies the above constraints from EPV and time-varying fine structure constant. If $\phi$ couples to the SM only through the trace of the energy momentum tensor, i.e.

$$
\mathcal{L}_{\phi}=d_{T} \frac{\phi}{M_{\mathrm{Pl}}} T_{\mu}^{\mu}
$$

we have

$$
d_{T}=d_{g}=d_{q}, \quad d_{\gamma}=0,
$$

therefore the observational bounds (2.10) and (2.14) are automatically satisfied. Note that the quintessence coupling to $T_{\mu}^{\mu}$ does not violate the equivalence principle, and also the time-varying fine structure constant applies for the low energy electromagnetic coupling which has a vanishing beta function, and therefore the corresponding $d_{\gamma}=0$ when the quintessence couplings take the form (2.15).

One may call the above type of quintessence a "metrical quintessence" since the specific coupling (2.15) can arise from the mixing of $\phi$ with the conformal factor of the spacetime metric $g_{\mu \nu}$ [30]. A specific such example is a theory which does not have any coupling between $\phi$ and the SM fields in an appropriate field basis, while having non-trivial couplings between $\phi$ and $g_{\mu \nu}$ through the $\phi$-dependent Planck mass. One can then move to the Einstein frame by making an appropriate Weyl transformation:

$$
g_{\mu \nu} \rightarrow \Omega(\phi) g_{\mu \nu}
$$

which would result in the quintessence coupling (2.15) in the Einstein frame. Yet, the coupling of metrical quintessence is constrained by the observational bounds on the deviation from the general relativity by the quintessence-mediated force in relativistic limit. For instance, from the measurement of the gravitational time delay effect to the Cassini spacecraft, one finds [34]

$$
d_{T}=d_{g}=d_{q}<3.4 \times 10^{-3}
$$




\section{De Sitter swampland conjecture for the pion and Higgs extrema}

As we have stressed, if the dS swampland conjecture (1.1) applies for the low energy quintessence potential (2.2) including the contributions from the electroweak symmetry and QCD chiral symmetry breaking, it is applicable also for the Higgs and QCD pseudoscalar meson potentials. In this section, we apply the dS swampland conjecture to some of the dS extrema of the pseudo-scalar meson or Higgs potential and examine its implications. For simplicity, we will take the simple effective field theory approach, assuming that all other degrees of freedom can be integrated out in such a way that the resulting effective theory is good enough over a field range including both the vacuum configuration and the relevant dS extrema, e.g. the entire field range of the pion field $\pi_{0} / f_{\pi} \in[0,2 \pi]$ and also the Higgs field range $\Delta H \sim v$.

Because we are considering both the vacuum solution and a dS extremum together, generically our results can receive corrections from the tadpoles or runaway behavior of the integrated scalar fields, which can be induced at the dS extremum point. We will see in the next section that those corrections do not significantly affect the results of this section when the observational constraints on the underlying dynamics are properly taken into account.

\subsection{Pion extremum}

To proceed, let us first consider the field configuration where the Higgs field is frozen at its vacuum value, $H=v(\phi)=m_{H}(\phi) / \sqrt{2 \lambda(\phi)}$, and integrate out all SM fields heavier than the QCD scale $\Lambda_{\mathrm{QCD}}$. The remained light scalar degrees of freedom are the quintessence field $\phi$ and the pseudo-scalar meson octet $\pi_{a}=(\pi, K, \eta)$ which correspond to the pseudoNambu-Goldstone bosons associated with the spontaneous breakdown of the QCD chiral symmetry:

$$
\mathrm{SU}(3)_{L} \times \mathrm{SU}(3)_{R} \rightarrow \mathrm{SU}(3)_{V} .
$$

The corresponding field manifold $\mathrm{SU}(3)_{L} \times \mathrm{SU}(3)_{R} / \mathrm{SU}(3)_{V}$ is compact and can be parametrized as

$$
U=\exp \left[i \frac{\pi_{a}}{f_{\pi}} \lambda_{a}\right]
$$

where $\lambda_{a}$ are the Gell-Mann matrices and $f_{\pi}$ is the pion decay constant. At leading order in chiral perturbation theory, the effective lagrangian of $U$ is given by

$$
\frac{f_{\pi}^{2}}{4} \operatorname{Tr}\left[\partial_{\mu} U^{\dagger} \partial^{\mu} U\right]+\frac{\Lambda^{3}}{2} \operatorname{Tr}\left[M_{q}\left(U+U^{\dagger}\right)\right],
$$

where $\Lambda$ can be identified as the condensation scale of the light quark fields, i.e. $\left\langle\bar{q}_{i} q_{j}\right\rangle=\Lambda^{3} \delta_{i j}$ for $q_{i}=(u, d, s)$, and $M_{q}=\operatorname{diag}\left(m_{u}, m_{d}, m_{s}\right)$ is the light quark mass matrix which is chosen to be real, diagonal and positive.

Because the meson field manifold $\mathrm{SU}(3)_{L} \times \mathrm{SU}(3)_{R} / \mathrm{SU}(3)_{V}$ is compact, there can be multiple dS extrema of the meson potential. Here, for simplicity we focus on the neutral 
pion $\pi_{0}$, while fixing all other mesons at their vacuum values. Then the effective potential of the pion and quintessence is given by

$$
V\left(\phi, \pi_{0} / f_{\pi}\right)=V_{\mathrm{eff}}(\phi)+\left(m_{u}(\phi)+m_{d}(\phi)\right) \Lambda^{3}(\phi)\left[1-\cos \left(\frac{\pi_{0}}{f_{\pi}(\phi)}\right)\right],
$$

where we choose the convention that $\left\langle\pi_{0}\right\rangle=0$ in the true vacuum, and the low energy QCD parameters $m_{u, d}, f_{\pi}$ and $\Lambda$ are understood to be generic functions of the quintessence field $\phi$. This potential is valid over the full range of the pion field $\pi_{0} / f_{\pi} \in[0,2 \pi]$ and has a dS local maximum along the pion direction at

$$
\frac{\pi_{0}}{f_{\pi}}=\pi
$$

Note that although we consider a leading order approximation in chiral perturbation theory, the periodicity of the pion field $\pi_{0} \equiv \pi_{0}+2 \pi f_{\pi}$ and the CP invariance under $\pi_{0} \rightarrow-\pi_{0}$ assure that this configuration is a dS local maximum of the exact pion potential up to negligible corrections due to the $\mathrm{CP}$ violating weak interactions. We then find

$$
\begin{aligned}
V\left(\phi, \pi_{0} / f_{\pi}=\pi\right) & =V_{\mathrm{eff}}(\phi)+2\left(m_{u}(\phi)+m_{d}(\phi)\right) \Lambda^{3}(\phi) \\
\nabla V\left(\phi, \pi_{0} / f_{\pi}\right. & =\pi)=V_{\mathrm{eff}}^{\prime}(\phi)+2\left(\frac{m_{u}^{\prime}+m_{d}^{\prime}}{m_{u}+m_{d}}+3 \frac{\Lambda^{\prime}}{\Lambda}\right)\left(m_{u}+m_{d}\right) \Lambda^{3},
\end{aligned}
$$

which results in

$$
M_{\mathrm{Pl}} \frac{\left|\nabla V\left(\phi, \pi_{0} / f_{\pi}=\pi\right)\right|}{V\left(\phi, \pi_{0} / f_{\pi}=\pi\right)}=\left|M_{\mathrm{Pl}}\left(\frac{m_{u}^{\prime}+m_{d}^{\prime}}{m_{u}+m_{d}}+\frac{3 \Lambda^{\prime}}{\Lambda}\right)+\mathcal{O}\left(10^{-43}\right)\right| \geq c,
$$

where we used the properties $(2.3)$ of $V_{\text {eff }}(\phi)$ yielding

$$
M_{\mathrm{Pl}} \frac{V_{\mathrm{eff}}^{\prime}}{\left(m_{u}+m_{d}\right) \Lambda^{3}} \lesssim \frac{V_{\mathrm{eff}}}{\left(m_{u}+m_{d}\right) \Lambda^{3}}=\frac{V_{\mathrm{eff}}}{m_{\pi}^{2} f_{\pi}^{2}} \sim 10^{-43},
$$

and applied the dS swampland conjecture (1.1) in the last step.

For us, it is most convenient to choose the renormalization scale of the light quark mass $m_{q}$ and the quark bilinear operator $\bar{q} q$ to be $\Lambda_{\mathrm{QCD}}$, for which

$$
\frac{\Lambda^{\prime}}{\Lambda}=\frac{\Lambda_{\mathrm{QCD}}^{\prime}}{\Lambda_{\mathrm{QCD}}}=\frac{d_{g}}{M_{\mathrm{Pl}}}, \quad \frac{m_{q}^{\prime}}{m_{q}}=\frac{d_{q}}{M_{\mathrm{Pl}}}
$$

where $d_{g}$ and $d_{q}$ are the low energy quintessence couplings defined in (2.7). Then the $\mathrm{dS}$ swampland conjecture applied for the pion and quintessence potential at the pion dS extremum results in

$$
c \lesssim \operatorname{Max}\left[d_{\tilde{q}}+3 d_{g}, \mathcal{O}\left(10^{-43}\right)\right]
$$

where

$$
d_{\tilde{q}}=\frac{m_{u} d_{u}+m_{d} d_{d}}{m_{u}+m_{d}} .
$$


We stress that the above bound is valid for arbitrary form of the quintessence potential and couplings.

Similarly to the case of the Higgs extremum discussed in [6], if $\phi$ is completely decoupled from the QCD sector, so that $d_{q}=d_{g}=0$, the parameter $c$ is required to be smaller than $V_{\text {eff }} / f_{\pi}^{2} m_{\pi}^{2} \sim 10^{-43}$, which is smaller than the conjectured value $c=\mathcal{O}(1)$ by many orders of magnitude. Again, by assuming appropriate form of couplings between the quintessence and the QCD sector, one can alleviate this bound on $c$ up to the value allowed by observational constraints. Then, for generic quintessence with $\left(d_{q}-d_{g}\right) /\left(d_{q}+d_{g}\right)=\mathcal{O}(1)$, the observational bound (2.10) on the violation of the equivalence principle (EP) can be applied to get

$$
c<2 \times 10^{-5} \text { for quintessence violating the EP. }
$$

On the other hand, for a metrical quintessence which couples to the SM only through the trace of energy momentum tensor and therefore has $d_{g}=d_{q}$, the bound on $c$ can be significantly relaxed. In such case, we can use the observational bound (2.18) on the deviation from the general relativity in the solar system to get

$$
c<1.4 \times 10^{-2} \text { for quintessence respecting the EP. }
$$

\subsection{Higgs extremum}

Let us now consider the Higgs dS extremum at $H=0$, which was discussed also in [6]. Here we will elaborate the discussion of [6] and examine if any useful bound on $c$ can be obtained from the consideration of the Higgs dS extremum. In the scalar field space near $H=0$, the effective potential can be written as

$$
V(H, \phi)=V_{\mathrm{eff}}(\phi)+\lambda(\phi) v^{4}(\phi)-\sum_{\psi}\left(y_{\psi}(\phi) H\left\langle\bar{\psi}_{L} \psi_{R}\right\rangle+h . c\right)-\frac{1}{2} m_{H}^{2}(\phi)|H|^{2}+\ldots,
$$

where we include the contribution from the quark condensations in the limit $H=0$ where all quarks are massless. To proceed, we can take the gauge that $H$ is identified as a real scalar field, and also choose the field basis where the Yukawa couplings $y_{\psi}$ are real, positive and diagonal. ${ }^{5}$ To avoid unnecessary complication due to nonzero tadpoles of the fields other than $\phi$, we then focus on the field configuration with

$$
\tilde{\pi}_{\psi}=\frac{\pi}{2}
$$

where $\tilde{\pi}_{\psi}$ denotes the phase of the quark condensation for $H=0$, i.e.

$$
\left\langle\bar{\psi}_{L} \psi_{R}\right\rangle_{H=0}=\tilde{\Lambda}^{3} e^{i \tilde{\pi}_{\psi}}
$$

for $\tilde{\Lambda}$ which corresponds to the QCD condensation scale for $H=0$, which is about an half of the QCD scale for $H=v$. For such field configuration, one immediately finds

$$
\partial_{H} V\left(H=0, \tilde{\pi}_{\psi}=\pi / 2\right)=\partial_{\tilde{\pi}_{\psi}} V\left(H=0, \tilde{\pi}_{\psi}=\pi / 2\right)=0,
$$

\footnotetext{
${ }^{5}$ The flavor changing weak interactions mediated by the W-boson in this field basis can be safely ignored.
} 
which results in [6]:

$$
M_{\mathrm{Pl}} \frac{|\nabla V|}{V}=M_{\mathrm{Pl}} \frac{\left|V_{\mathrm{eff}}^{\prime}+\left(\lambda^{\prime} / \lambda+4 v^{\prime} / v\right) \lambda v^{4}\right|}{V_{\mathrm{eff}}(\phi)+\lambda(\phi) v(\phi)^{4}}=\left|M_{\mathrm{Pl}}\left(\frac{\lambda^{\prime}}{\lambda}+\frac{4 v^{\prime}}{v}\right)+\mathcal{O}\left(10^{-55}\right)\right| \gtrsim c .
$$

Translating the above result to an observational bound on $c$ is more complicated and model-dependent than the case of the pion extremum. Yet, with the matching condition (2.9) on $d_{g}$ including the relevant radiative corrections and also the tree level matching condition $d_{q} / M_{\mathrm{Pl}}=m_{q}^{\prime} / m_{q}=y_{q}^{\prime} / y_{q}+v^{\prime} / v$, we can estimate the maximal value of $c$ compatible with the observational constraints on the low energy quintessence couplings. Given the observational bounds on $d_{g}$ and $d_{q}$, the maximal value of $c$ can be achieved when $\left|\lambda^{\prime} / \lambda\right| \gg\left|v^{\prime} / v\right|,\left|y_{q}^{\prime} / y_{q}\right|$ and $\lambda^{\prime} / \lambda$ saturates the bound on $d_{g}$ through the two loop contribution represented by the last term of (2.9). In fact, the model discussed in [6] corresponds to such case as it assumes $v^{\prime}=y_{q}^{\prime}=0$ with $\lambda^{\prime} \neq 0$. Inserting all the involved numerical factors, we find that the corresponding bound on $c$ is given by

$$
c \leq \operatorname{Max}\left[1.5 \times 10^{4} d_{g}, 10^{-55}\right] \lesssim 4.4 \times 10^{-2}
$$

which is significantly weaker than the bound (3.12) from the pion extremum. Note that for a metrical quintessence, we have $\left|\lambda^{\prime} / \lambda\right| \ll\left|v^{\prime} / v\right|$ and the bound on $c$ from the Higgs extremum is same as the one from the pion extremum, i.e. $c<1.4 \times 10^{-2}$.

\section{Effects of the tadpole or runaway of additional scalar fields}

In the previous section, we discussed the implications of the pion or Higgs extremum for the dS swampland conjecture within an effective theory while assuming that other scalar degrees of freedom can be integrated out in such a way that the resulting effective theory can describe well the relevant low energy physics over the entire field range of the pion field, i.e. $\pi_{0} / f_{\pi} \in[0,2 \pi]$, and also over the Higgs field range $\Delta H \sim v$. Here we examine possible effects of the tadpole or runaway behavior of the integrated scalar fields, which can be induced at the pion or Higgs dS extremum. As it provides the most stringent bound on $c$, we will focus on the case of the pion extremum. As we will see, the bounds on $c$ obtained in section 3 can not be significantly relaxed by additional scalar fields when the observational constraints are properly taken into account.

Let $\Phi$ denote a generic scalar field which is integrated out in the effective potential (3.4). As the quintessence is the only rolling field in the present Universe, $\Phi$ should be properly stabilized at least when the pion field is at the vacuum with $\pi_{0} / f_{\pi}=0$. Then, one can always choose $\langle\Phi\rangle_{\pi_{0}=0}=0$ and expand the full potential of $\phi, \pi_{0}$ and $\Phi$ as follows:

$$
V\left(\phi, \pi_{0}, \Phi\right)=V_{\mathrm{eff}}(\phi)+V_{\mathrm{up}}\left(\phi, \pi_{0}\right)+\frac{1}{2} m_{\Phi}^{2}(\phi) \Phi^{2}+\left(\frac{\Phi}{2 \Lambda_{\Phi}(\phi)}+\cdots\right) V_{\mathrm{up}}\left(\phi, \pi_{0}\right),
$$

where

$$
\begin{aligned}
V_{\mathrm{up}}\left(\phi, \pi_{0}\right) & \equiv V\left(\phi, \pi_{0}, \Phi=0\right)-V\left(\phi, \pi_{0}=0, \Phi=0\right) \\
& \simeq\left(m_{u}(\phi)+m_{d}(\phi)\right) \Lambda^{3}(\phi)\left(1-\cos \frac{\pi_{0}}{f_{\pi}(\phi)}\right)=m_{\pi}^{2}(\phi) f_{\pi}^{2}(\phi)\left(1-\cos \frac{\pi_{0}}{f_{\pi}(\phi)}\right)
\end{aligned}
$$


and the ellipsis denotes the terms higher order in $\Phi$. Obviously, here $m_{\Phi}$ is the mass of $\Phi$ when $\pi_{0} / f_{\pi}=0$, and $\Lambda_{\Phi}$ is a mass scale parametrizing the coupling of $\Phi$ to the pions or more generically to the low energy QCD sector.

If $m_{\Phi} \Lambda_{\Phi}>m_{\pi} f_{\pi}$, $\Phi$ is stabilized with a small field shift even when the pion field is at $\pi_{0} / f_{\pi}=\pi$. The corresponding tadpole is determined by

$$
\partial_{\pi_{0}} V\left(\phi, \pi_{0} / f_{\pi}=\pi, \Phi=\delta \Phi\right)=\partial_{\Phi} V\left(\phi, \pi_{0} / f_{\pi}=\pi, \Phi=\delta \Phi\right)=0
$$

yielding

$$
\frac{\delta \Phi}{\Lambda_{\Phi}} \simeq \frac{m_{\pi}^{2} f_{\pi}^{2}}{m_{\Phi}^{2} \Lambda_{\Phi}^{2}}
$$

One may then apply the dS swampland conjecture for the shifted extremum point, which would result in

$$
\begin{aligned}
& M_{\mathrm{Pl}} \frac{\left|\nabla V\left(\phi, \pi_{0} / f_{\pi}=\pi, \Phi=\delta \Phi\right)\right|}{V\left(\phi, \pi_{0} / f_{\pi}=\pi, \Phi=\delta \Phi\right)}=M_{\mathrm{Pl}} \frac{\left|\partial_{\phi} V\left(\phi, \pi_{0} / f_{\pi}=\pi, \Phi=\delta \Phi\right)\right|}{V\left(\phi, \pi_{0} / f_{\pi}=\pi, \Phi=\delta \Phi\right)} \\
& \quad \simeq M_{\mathrm{Pl}}\left|\partial_{\phi} \ln V_{\mathrm{up}}\left(\phi, \pi_{0} / f_{\pi}=\pi\right)-\frac{m_{\pi}^{2} f_{\pi}^{2}}{m_{\Phi}^{2} \Lambda_{\Phi}^{2}} \partial_{\phi} \ln \left(\frac{V_{\mathrm{up}}\left(\phi, \pi_{0} / f_{\pi}=\pi\right)}{m_{\Phi}^{2}(\phi) \Lambda_{\Phi}^{2}(\phi)}\right)\right| \\
& \quad=M_{\mathrm{Pl}}\left|\left(\frac{m_{u}^{\prime}+m_{d}^{\prime}}{m_{u}+m_{d}}+3 \frac{\Lambda^{\prime}}{\Lambda}\right)-\frac{m_{\pi}^{2} f_{\pi}^{2}}{m_{\Phi}^{2} \Lambda_{\Phi}^{2}}\left(\frac{m_{u}^{\prime}+m_{d}^{\prime}}{m_{u}+m_{d}}+3 \frac{\Lambda^{\prime}}{\Lambda}-\partial_{\phi} \ln \left(m_{\Phi}^{2} \Lambda_{\Phi}^{2}\right)\right)\right| \\
& \quad=\left|\left(d_{\tilde{q}}+3 d_{g}\right)\left(1-\frac{m_{\pi}^{2} f_{\pi}^{2}}{m_{\Phi}^{2} \Lambda_{\Phi}^{2}}\right)+\frac{m_{\pi}^{2} f_{\pi}^{2}}{m_{\Phi}^{2} \Lambda_{\Phi}^{2}} M_{\mathrm{Pl}} \partial_{\phi} \ln \left(m_{\Phi}^{2} \Lambda_{\Phi}^{2}\right)\right| \geq c .
\end{aligned}
$$

Note that the terms suppressed by $m_{\pi}^{2} f_{\pi}^{2} / m_{\Phi}^{2} \Lambda_{\Phi}^{2}$ correspond to the corrections to eq. (3.7) in section 3, which arise from the tadpole of $\Phi$ induced at $\pi_{0} / f_{\pi}=\pi$.

Let us apply the above results for the SM scalar degrees of freedom which are either elementary or composite. Fist of all, for $\Phi$ being the pseudo-scalar mesons such as $K$ and $\eta, \mathrm{P}$ or CP symmetry assures that the linear coupling of $\Phi$ to $V_{\mathrm{up}}$ is highly suppressed, e.g. $\Lambda_{\Phi} \gg v$, and therefore $f_{\pi}^{2} m_{\pi}^{2} / m_{\Phi}^{2} \Lambda_{\Phi}^{2} \ll 10^{-5}$. For $\Phi$ being the SM Higgs boson, we have $\Lambda_{\Phi} \sim v$ and again $m_{\pi}^{2} f_{\pi} / m_{\Phi}^{2} \Lambda_{\Phi}^{2} \sim m_{\pi}^{2} f_{\pi}^{2} / m_{h}^{2} v^{2} \ll 10^{-5}$. This assures the possible corrections due to the tadpole of the pseudo-scalar mesons and Higgs boson are much smaller than the observational bound on $d_{g}$ and $d_{q}$, and therefore can be safely ignored. In fact, the only scalar degree of freedom of the SM which can have a non-negligible value of $f_{\pi}^{2} m_{\pi}^{2} / m_{\Phi}^{2} \Lambda_{\Phi}^{2}$ is the quark-antiquark composite scalar $\sigma$ which controls the size of the light quark condensation: ${ }^{6}$

$$
\langle\bar{q} q\rangle \propto e^{-\sigma / \Lambda_{\sigma}},
$$

for which

$$
m_{\sigma} \sim \Lambda_{\sigma} \sim \Lambda_{\mathrm{QCD}}
$$

\footnotetext{
${ }^{6} \mathrm{In}$ real QCD, $\sigma$ has a too broad decay width, so there is no corresponding particle state. However, yet $\sigma$ can be relevant for the dS swampland conjecture as the potential energy varies as a function of $\sigma$.
} 
In this case, the corresponding suppression factor $m_{\pi}^{2} f_{\pi}^{2} / m_{\sigma}^{2} \Lambda_{\sigma}^{2} \sim m_{q} / \Lambda_{\mathrm{QCD}}$ is not small enough. However the accompanying factor which is given by

$$
M_{\mathrm{Pl}} \partial_{\phi} \ln \left(m_{\sigma}^{2} \Lambda_{\sigma}^{2}\right)=4 M_{\mathrm{Pl}} \frac{\Lambda_{\mathrm{QCD}}^{\prime}}{\Lambda_{\mathrm{QCD}}}=4 d_{g}
$$

provides additional suppression, so that again the tadpole of $\sigma$ at the pion extremum does not alter the result (3.7).

Our discussion above suggests that the upper bound on $c$ can be relaxed if there exists some scalar field $\Phi$ (other than those in the SM) with $\frac{m_{\pi}^{2} f_{\pi}^{2}}{m_{\Phi}^{2} \Lambda_{\Phi}^{2}} M_{\mathrm{Pl}} \partial_{\phi} \ln \left(m_{\Phi}^{2} \Lambda_{\Phi}^{2}\right) \gg \mathcal{O}\left(10^{-5}\right.$ $10^{-2}$ ) at $\pi_{0} / f_{\pi}=\pi$, i.e. a light scalar which has a sizable tadpole or runaway behavior at the pion extremum. In string theory, the most promising candidate for such scalar field is a light modulus $\chi$. Even when $\chi$ has a sizable tadpole or runaway behavior at $\pi_{0} / f_{\pi}=\pi$, it has to be stabilized at certain vacuum value $\langle\chi\rangle$ with a mass $m_{\chi}>H_{0} \sim 10^{-33} \mathrm{eV}$ at $\pi_{0} / f_{\pi}=0$. Again one can choose a field basis for which $\langle\chi\rangle=0$ at $\pi_{0} / f_{\pi}=0$, and then the potential can be expanded as

$$
V\left(\phi, \pi_{0}, \chi\right)=V_{\mathrm{eff}}(\phi)+V_{\mathrm{up}}\left(\phi, \pi_{0}\right)+\frac{1}{2} m_{\chi}^{2}(\phi) \chi^{2}+\left(c_{\chi} \frac{\chi}{2 M_{\mathrm{Pl}}}+\cdots\right) V_{\mathrm{up}}\left(\phi, \pi_{0}\right),
$$

where we introduce a dimensionless parameter $c_{\chi}$ to parametrize the coupling of $\chi$ to the low energy QCD sector. Here the coupling $c_{\chi}$ should be the low energy consequence of the modulus couplings to the QCD sector, which can be parametrized as

$$
\mathcal{L}_{\chi}=-\tilde{d}_{g} \frac{\chi}{M_{\mathrm{Pl}}} \frac{\beta_{s}}{2 g_{s}} G^{i \mu \nu} G_{\mu \nu}^{i}-\sum_{q=u, d, s}\left(\tilde{d}_{q}+\gamma_{m} \tilde{d}_{g}\right) m_{q} \frac{\chi}{M_{\mathrm{Pl}}} \bar{q},
$$

and then

$$
c_{\chi}=\frac{m_{u} \tilde{d}_{u}+m_{d} \tilde{d}_{d}}{m_{u}+m_{d}}+3 \tilde{d}_{g} \equiv \tilde{d}_{\tilde{q}}+3 \tilde{d}_{g} .
$$

There are some range of $m_{\chi}$ for which the modulus $\chi$ is obviously in conflict with the observational constraints or not useful for relaxing the bound on $c$. For instance, for a relatively massive $\chi$ with

$$
m_{\chi} \gtrsim 1.5 \times 10^{-9} c_{\chi} \mathrm{eV},
$$

one can apply (4.5) with $m_{\Phi}=m_{\chi}$ and $\Lambda_{\Phi}=M_{\mathrm{Pl}} / c_{\chi}$ to ensure that the modulus tadpole $\delta \chi$ is small enough to keep the bound (3.10) unaffected. Also, the following mass regions are excluded by the blackhole superradiance [46]:

$$
\begin{aligned}
5 \times 10^{-13} \mathrm{eV} & \lesssim m_{\chi} \\
10^{-17} \mathrm{eV} & \lesssim m_{\chi} \lesssim 6 \times 10^{-11} \mathrm{eV}, \\
8 \times 10^{-17} \mathrm{eV}, & \lesssim m_{\chi} \lesssim 10^{-18} \mathrm{eV} .
\end{aligned}
$$

If $\chi$ is light enough, e.g.

$$
m_{\chi}<5 \times 10^{-12} c_{\chi}^{1 / 2} \mathrm{eV}
$$


the resulting modulus tadpole $\delta \chi$ is of $\mathcal{O}\left(M_{\mathrm{Pl}}\right)$, so a nearby stationary point is not guaranteed to exist. In such case, one can apply the dS swampland conjecture to the field configuration with $\pi_{0} / f_{\pi}=\pi$ and $\chi=0$, rather than the configuration with $\pi_{0} / f_{\pi}=\pi$ and $\chi=\delta \chi$. This then leads to a new upper bound on $c$ as

$$
\begin{aligned}
M_{\mathrm{Pl}} \frac{\left|\nabla V\left(\phi, \pi_{0} / f_{\pi}=\pi, \chi=0\right)\right|}{V\left(\phi, \pi_{0} / f_{\pi}=\pi, \chi=0\right)} & =M_{\mathrm{Pl}} \frac{\sqrt{\left(\partial_{\phi} V\right)^{2}+\left(\partial_{\chi} V\right)^{2}}}{V} \\
& \simeq \sqrt{\left(d_{\tilde{q}}+3 d_{g}\right)^{2}+c_{\chi}^{2}} \geq c .
\end{aligned}
$$

For $m_{\chi}<10^{-18} \mathrm{eV}$, the modulus couplings $\tilde{d}_{g}$ and $\tilde{d}_{q}$ are constrained as the quintessence couplings $d_{q}$ and $d_{g}$ by both the non-observation of the violation of the equivalence principle [36] and tests of general relativity in the solar system [34, 42-45]. Then the above new bound is essentially equivalent to the bound (3.10), which means that a ultralight modulus with $m_{\chi}<10^{-18} \mathrm{eV}$ is not useful for relaxing the bound (3.10). If $\left(\tilde{d}_{g}-\tilde{d}_{q}\right) /\left(\tilde{d}_{g}+\tilde{d}_{q}\right)=\mathcal{O}(1)$, so that the modulus couplings violate the equivalence principle, one can use the corresponding bounds on $\tilde{d}_{g}$ and $\tilde{d}_{q}$ to get the following bound on $c$ from (4.15):

$$
c<2 \times 10^{-5} \text { for } m_{\chi}<10^{-13} \mathrm{eV} \text {. }
$$

This means that a modulus with $m_{\chi}<10^{-13} \mathrm{eV}$ and $\left(\tilde{d}_{g}-\tilde{d}_{q}\right) /\left(\tilde{d}_{g}+\tilde{d}_{q}\right)=\mathcal{O}(1)$ is again not useful for relaxing the bound (3.10).

The light modulus $\chi$ which may have a sizable tadpole or runaway behavior at the pion extremum is constrained also by the cosmological modulus mass density generated by the modulus misalignment $\Delta \chi$ in the early Universe which is induced by the coupling $c_{\chi}$. Note that a nonzero $c_{\chi}$ means that $\chi$ couples to the gluons and/or the light quarks, so the thermal free energy of gluons and light quarks in the early Universe depends on $\chi$. Such modulusdependent free energy induces a modulus misalignment, which eventually produces the modulus dark matter. To examine this issue, let us consider the finite temperature effective potential before the QCD phase transition, which is given by

$$
V(\chi, T)=-\frac{\pi^{2}}{90} g_{*}(T) T^{4}+\frac{2 T^{2}}{3}\left(3+\frac{N_{f}}{2}\right) \frac{g_{s}^{2}(\chi) T^{2}}{6}+\sum_{m_{q}<T} \frac{T^{2}}{4}\left(m_{q}^{2}(\chi)+\frac{g_{s}^{2}(\chi) T^{2}}{6}\right),
$$

where $g_{*}$ is the number of degrees of freedom contributing to the free energy and $N_{f}$ is the number of quark flavors lighter than $T$. This potential provides a slope to the modulus $\chi$ as

$$
\frac{\partial V}{\partial \chi}=\frac{2 \alpha_{s}^{2} T^{4}}{9}\left(3+\frac{7}{8} N_{f}\right)\left(b_{3} \frac{\tilde{d}_{g}}{M_{\mathrm{Pl}}}-\sum_{\Lambda_{\mathrm{QCD}}<m_{q}<T} \frac{2}{3} \partial_{\chi} \ln \left(y_{q} v\right)\right)+\sum_{m_{q}<T} \frac{\tilde{d}_{q} m_{q}^{2} T^{2}}{2 M_{\mathrm{Pl}}},
$$

which induces a modulus misalignment

$$
\frac{\Delta \chi}{M_{\mathrm{Pl}}} \simeq \frac{\partial_{\chi} V}{M_{\mathrm{Pl}} H^{2}}
$$




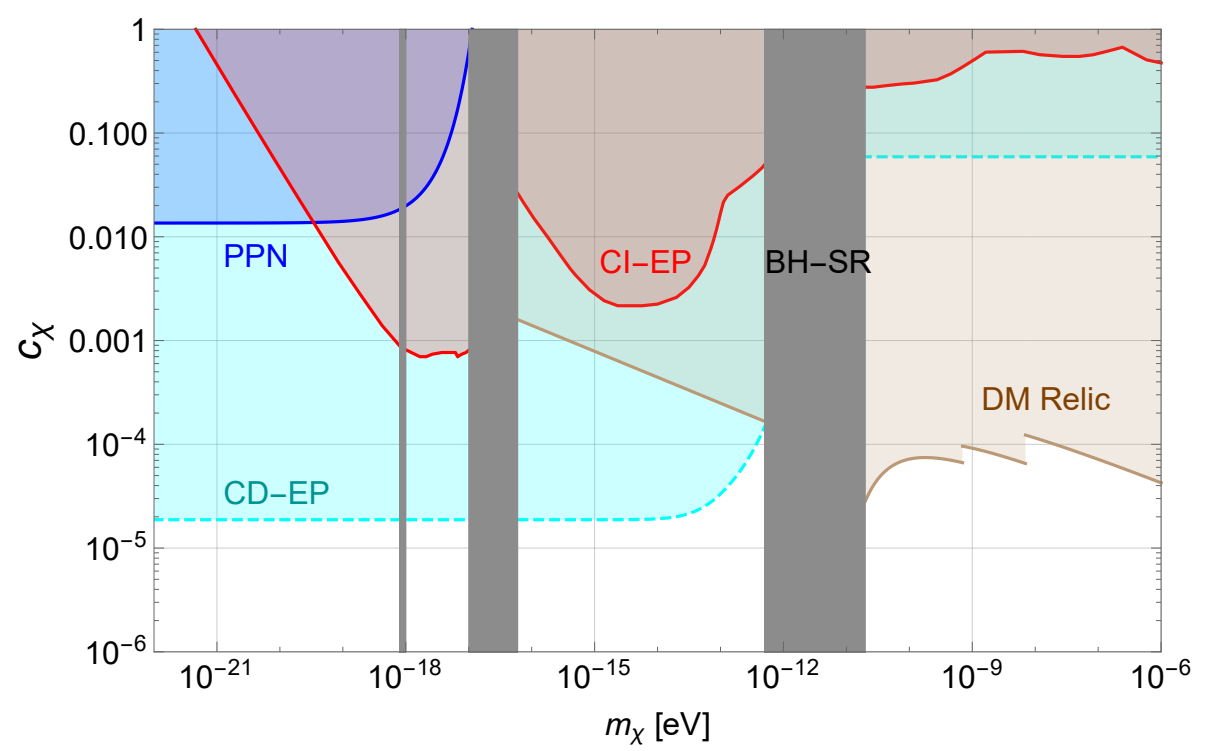

Figure 1. Observational upper bound on the modulus coupling $c_{\chi}=\tilde{d}_{\tilde{q}}+3 \tilde{d}_{g}$ as a function of the modulus mass $m_{\chi}$. The gray regions are excluded by the blackhole superradiance (BH-SR) [46]. The cyan region and the red region are excluded, respectively, by the composition-dependent $[37,47]$ and composition-independent [48, 49] equivalence principle (CD-EP and CI-EP) tests. Constraints on the parametrized post-Newtonian (PPN) parameters [34, 42-45] exclude the blue region, which applies not only for a generic modulus, but also for a metrical modulus respecting the equivalence principle. The dark matter relic abundance constrains the modulus misalignment, excluding the brown region.

We estimate such modulus misalignment at $T=T_{\chi} \simeq \sqrt{m_{\chi} M_{\mathrm{Pl}}}$ and find

$$
\begin{aligned}
& \frac{\Delta \chi}{M_{\mathrm{Pl}}} \gtrsim 0.5 \frac{\left(3+\frac{7}{8} N_{f}\right)\left(11-\frac{2}{3} N_{f}\right)}{g_{*}\left(T_{\chi}\right)} \alpha_{s}^{2}\left(T_{\chi}\right) c_{\chi}+\sum_{m_{q}>T_{\chi}} \frac{0.4}{\sqrt{g_{*}\left(m_{q}\right)}} c_{\chi} \quad \text { for } m_{\chi}>10^{-11} \mathrm{eV} \\
& \frac{\Delta \chi}{M_{\mathrm{Pl}}} \gtrsim 0.4 c_{\chi} \quad \text { for } \quad m_{\chi} \ll 10^{-11} \mathrm{eV}
\end{aligned}
$$

where for simplicity $\tilde{d}_{q}$ and $\tilde{d}_{g}$ are assumed to have a similar value. Requiring that the resulting modulus mass density does not exceed the observed dark matter mass density, i.e.

$$
\Omega_{\chi} h^{2}<0.12,
$$

we obtain the following bounds on the modulus coupling:

$$
\begin{aligned}
c_{\chi} \lesssim \frac{2.2 \times 10^{-6}}{\alpha_{s}^{2}\left(T_{\chi}\right) g_{*}^{-1}\left(T_{\chi}\right)+\sum_{m_{q}>T_{\chi}} 0.015 g_{*}^{-1 / 2}\left(m_{q}\right)}\left(\frac{10^{-12} \mathrm{eV}}{m_{\chi}}\right)^{\frac{1}{4}} \text { for } m_{\chi}>10^{-11} \mathrm{eV}, \\
c_{\chi} \lesssim 1.4 \times 10^{-4}\left(\frac{10^{-12} \mathrm{eV}}{m_{\chi}}\right)^{\frac{1}{4}} \text { for } m_{\chi} \ll 10^{-11} \mathrm{eV} .
\end{aligned}
$$

In figure 1, we summarize the available constraints on the modulus coupling $c_{\chi}$ as a function of $m_{\chi}$. The blackhole superradiance (BH-SR) [46] excludes the modulus mass 


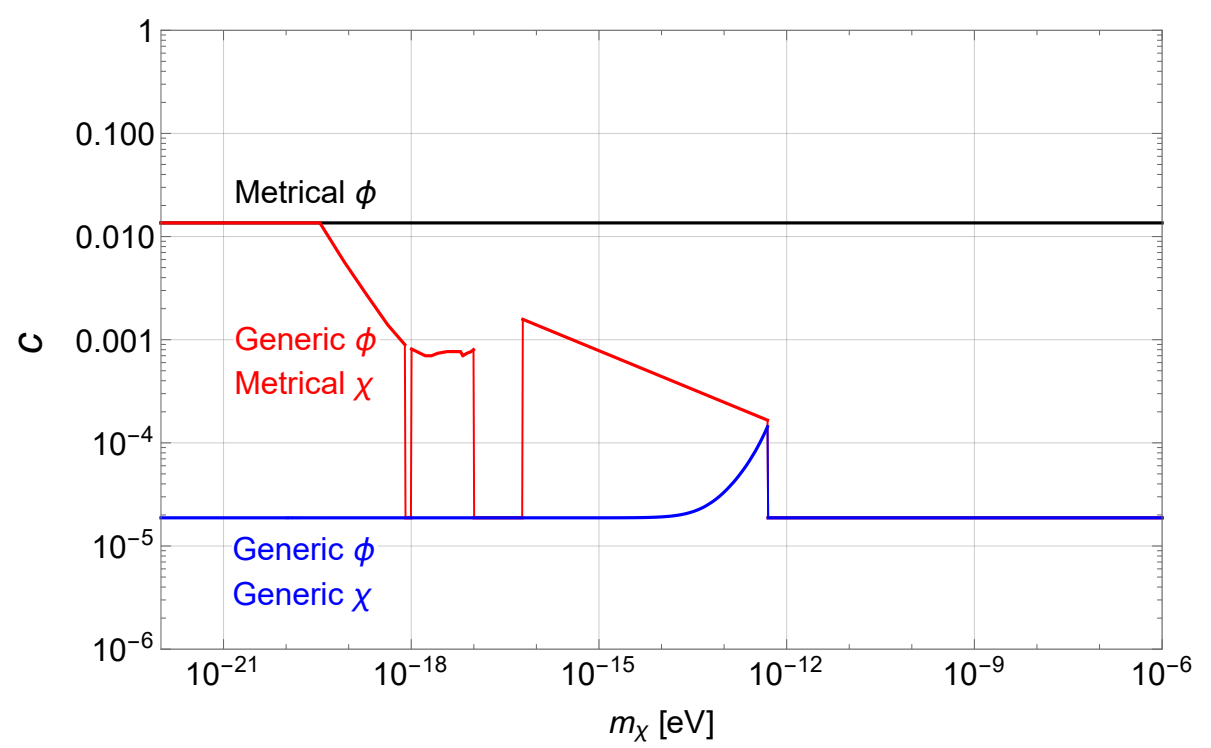

Figure 2. The refined upper bound on the parameter $c$ as a function of the mass $m_{\chi}$ of a moduluslike scalar field $\chi$ introduced to relax the original bounds (3.12) and (3.13). This shows that the original bounds are not significantly relaxed by additional light scalar once the observational constraints on such light scalar are properly taken into account.

range (4.13) which corresponds to the gray region in figure 1 . The cyan region bounded by a dotted line for $m_{\chi}<10^{-12} \mathrm{eV}$ is excluded by the MICROSCOPE test of the compositiondependent equivalence principle (CD-EP) [37], while the region above $10^{-12} \mathrm{eV}$ is excluded by the short range test of the CD-EP [47]. If $\chi$ respects the equivalence principle, e.g. a metrical modulus $\chi$ with $\tilde{d}_{q}=\tilde{d}_{g}$, the CD-EP bounds do not apply anymore. Yet, for certain range of $m_{\chi}$, such metrical modulus can result in an observable deviation of the gravitational potential from $1 / r$, which is bounded by the composition-independent equivalence principle (CI-EP) test. The red region of figure 1 is excluded by such experiments testing the CI-EP $[48,49]$. The blue region of figure 1 is excluded by the measurement of the gravitational time delay to photons from the Cassini spacecraft in the parametrized post-Newtonian (PPN) formalism [34, 42-45], which applies not only for generic modulus, but also for a metrical modulus which respects the equivalence principle. Finally, the brown region is excluded by the bound on the relic modulus dark matter produced by the modulus misalignment induced by the coupling $c_{\chi}$.

Since its coupling $c_{\chi}$ is severely constrained as above, the modulus-like scalar $\chi$ can not significantly relax the bounds (3.12) and (3.13) which were obtained in the effective theory where $\chi$ is integrated out. In figure 2, we depict the refined bound on $c$ taking into account the effects of $\chi$ for three different cases. The blue line is the bound for the case that both the quintessence $\phi$ and the additional modulus $\chi$ have generic couplings violating the equivalence principle, while the black (red) line corresponds to the case that $\phi(\chi)$ respects the equivalence principle. The results of figure 2 can be extrapolated to $m_{\chi} \ll 10^{-21} \mathrm{eV}$ in a straightforward manner. On the other hand, for $m_{\chi}>10^{-6} \mathrm{eV}$, one can use (4.4) and (4.5) to assure that the bounds (3.12) and (3.13) can not be relaxed by introducing $\chi$. 
With the above results, let us now regard (3.12) as the bound on $c$ when both $\phi$ and $\chi$ have generic couplings to violate the equivalence principle, while (3.12) corresponds to the bound when any of $\phi$ and $\chi$ respects the equivalence principle. Then our results imply that those bounds on $c$ can not be significantly relaxed by additional modulus-like light scalar field once the observational constraints on such scalar field are properly taken into account. ${ }^{7}$

\section{Conclusion}

In this paper we examined the implications of the pion or Higgs dS extrema for the dS swampland conjecture, while focusing on the possible (model-independent or modeldependent) bound on the parameter $c$. Applying the dS swampland conjecture to the pion extremum at $\cos \left(\pi_{0} / f_{\pi}\right)=-1$, we could derive a model-independent upper bound on $c$ given in terms of the low energy quintessence couplings.

If the quintessence couplings take a rather specific form to respect the equivalence principle, which would be the case when the quintessence couples to the SM sector only through the trace of the energy momentum tensor, $c$ is bounded essentially by the observational bound on the deviation from the general relativity by the quintessence-mediated force in relativistic limit, yielding $c<1.4 \times 10^{-2}$. However, for generic quintessence whose couplings violate the equivalence principle, the parameter $c$ is more strongly bounded as $c<2 \times 10^{-5}$ by the non-observation of the violation of the equivalence principle in nonrelativistic limit. These bounds on $c$ are rather robust as (i) they are obtained within the framework of the most general quintessence potential and couplings and (ii) they can not be significantly relaxed by an additional light scalar field which may have a nonzero tadpole or runaway behavior at the pion dS extremum, if the observational constraints on such light scalar field are properly taken into account. One can do a similar analysis for the Higgs extremum at $H=0$, but the resulting bound $c \lesssim 4.4 \times 10^{-2}$ is weaker than those from the pion extremum as the quintessence couplings to the Higgs sector is more weakly constrained than those to the low energy QCD sector.

Our bounds on $c$ from the pion extremum appear to have a significant tension with the dS swampland conjecture (1.1) which assumes $c=\mathcal{O}(1)$. Yet the conjecture (1.1) can be modified or refined, for instance as in $[7,8]$ and [9], which would allow us to avoid the bounds from the Higgs and pion extrema. If it is the right direction to pursue, our results provide additional motivation for such refinement of the conjecture.

\section{Acknowledgments}

This work was supported by IBS under the project code IBS-R018-D1. K.C. thanks A. Hebecker for useful discussions. C.S.S. thanks Deog Ki Hong for valuable discussions during the CERN-Korea TH Institute, and also the CERN Theory Group for the hospitality.

\footnotetext{
${ }^{7}$ Our result suggests that the stronger bound (3.12) can be relaxed by about one order of magnitude by a modulus $\chi$ with $m_{\chi}=\mathcal{O}\left(10^{-13}\right) \mathrm{eV}$. However this minor point does not change the main message of our results.
} 
Open Access. This article is distributed under the terms of the Creative Commons Attribution License (CC-BY 4.0), which permits any use, distribution and reproduction in any medium, provided the original author(s) and source are credited.

\section{References}

[1] G. Obied, H. Ooguri, L. Spodyneiko and C. Vafa, de Sitter Space and the Swampland, arXiv: 1806.08362 [INSPIRE].

[2] P. Agrawal, G. Obied, P.J. Steinhardt and C. Vafa, On the Cosmological Implications of the String Swampland, Phys. Lett. B 784 (2018) 271 [arXiv:1806.09718] [InSPIRE].

[3] B. Ratra and P.J.E. Peebles, Cosmological Consequences of a Rolling Homogeneous Scalar Field, Phys. Rev. D 37 (1988) 3406 [InSPIRE].

[4] C. Wetterich, Cosmology and the Fate of Dilatation Symmetry, Nucl. Phys. B 302 (1988) 668 [arXiv: 1711.03844] [INSPIRE].

[5] I. Zlatev, L.-M. Wang and P.J. Steinhardt, Quintessence, cosmic coincidence and the cosmological constant, Phys. Rev. Lett. 82 (1999) 896 [astro-ph/9807002] [InSPIRE].

[6] F. Denef, A. Hebecker and T. Wrase, de Sitter swampland conjecture and the Higgs potential, Phys. Rev. D 98 (2018) 086004 [arXiv: 1807.06581] [INSPIRE].

[7] G. Dvali and C. Gomez, On Exclusion of Positive Cosmological Constant, arXiv: 1806.10877 [INSPIRE].

[8] D. Andriot, On the de Sitter swampland criterion, Phys. Lett. B 785 (2018) 570 [arXiv: 1806.10999] [INSPIRE].

[9] S.K. Garg and C. Krishnan, Bounds on Slow Roll and the de Sitter Swampland, arXiv: 1807.05193 [INSPIRE].

[10] S. Banerjee, U. Danielsson, G. Dibitetto, S. Giri and M. Schillo, Emergent de Sitter cosmology from decaying $A d S$, arXiv:1807.01570 [INSPIRE].

[11] L. Aalsma, M. Tournoy, J.P. Van Der Schaar and B. Vercnocke, Supersymmetric embedding of antibrane polarization, Phys. Rev. D 98 (2018) 086019 [arXiv: 1807.03303] [INSPIRE].

[12] A. Achúcarro and G.A. Palma, The string swampland constraints require multi-field inflation, arXiv: 1807.04390 [INSPIRE].

[13] J.-L. Lehners, Small-Field and Scale-Free: Inflation and Ekpyrosis at their Extremes, JCAP 11 (2018) 001 [arXiv:1807.05240] [INSPIRE].

[14] A. Kehagias and A. Riotto, A note on Inflation and the Swampland, arXiv:1807.05445 [INSPIRE].

[15] M. Dias, J. Frazer, A. Retolaza and A. Westphal, Primordial Gravitational Waves and the Swampland, arXiv: 1807.06579 [INSPIRE].

[16] E.Ó. Colgáin, M.H. P.M. Van Putten and H. Yavartanoo, Observational consequences of $H_{0}$ tension in de Sitter Swampland, arXiv:1807.07451 [INSPIRE].

[17] C. Roupec and T. Wrase, de Sitter extrema and the swampland, arXiv:1807.09538 [INSPIRE].

[18] D. Andriot, New constraints on classical de Sitter: flirting with the swampland, arXiv: 1807.09698 [INSPIRE]. 
[19] H. Matsui and F. Takahashi, Eternal Inflation and Swampland Conjectures, arXiv:1807.11938 [INSPIRE].

[20] I. Ben-Dayan, Draining the Swampland, arXiv:1808.01615 [INSPIRE].

[21] C. Damian and O. Loaiza-Brito, Two-field axion inflation and the swampland constraint in the flux-scaling scenario, arXiv:1808.03397 [INSPIRE].

[22] J.P. Conlon, The de Sitter swampland conjecture and supersymmetric AdS vacua, Int. J. Mod. Phys. A 33 (2018) 1850178 [arXiv: 1808.05040] [INSPIRE].

[23] W.H. Kinney, S. Vagnozzi and L. Visinelli, The Zoo Plot Meets the Swampland: Mutual (In)Consistency of Single-Field Inflation, String Conjectures and Cosmological Data, arXiv: 1808.06424 [INSPIRE].

[24] K. Dasgupta, M. Emelin, E. McDonough and R. Tatar, Quantum Corrections and the de Sitter Swampland Conjecture, arXiv:1808.07498 [INSPIRE].

[25] S. Kachru and S.P. Trivedi, A comment on effective field theories of flux vacua, arXiv: 1808.08971 [INSPIRE].

[26] L. Heisenberg, M. Bartelmann, R. Brandenberger and A. Refregier, Dark Energy in the Swampland II, arXiv:1809.00154 [INSPIRE].

[27] H. Murayama, M. Yamazaki and T.T. Yanagida, Do We Live in the Swampland?, arXiv: 1809.00478 [INSPIRE].

[28] M.C.D. Marsh, The Swampland, Quintessence and the Vacuum Energy, arXiv:1809.00726 [INSPIRE].

[29] K. Choi, String or M-theory axion as a quintessence, Phys. Rev. D 62 (2000) 043509 [hep-ph/9902292] [INSPIRE].

[30] K. Choi, Quintessence, flat potential and string/M theory axion, in Supersymmetry, supergravity and superstring. Proceedings, KIAS-CTP International Symposium, Seoul, Korea, June 23-26, 1999, pp. 280-299 (1999) [hep-ph/9912218] [INSPIRE].

[31] C.-I. Chiang and H. Murayama, Building Supergravity Quintessence Model, arXiv: 1808.02279 [INSPIRE].

[32] M. Cicoli, S. De Alwis, A. Maharana, F. Muia and F. Quevedo, de Sitter vs Quintessence in String Theory, arXiv:1808.08967 [INSPIRE].

[33] Y. Akrami, R. Kallosh, A. Linde and V. Vardanyan, The landscape, the swampland and the era of precision cosmology, arXiv: 1808.09440 [INSPIRE].

[34] B. Bertotti, L. Iess and P. Tortora, A test of general relativity using radio links with the Cassini spacecraft, Nature 425 (2003) 374 [INSPIRE].

[35] R.M. Godun et al., Frequency Ratio of Two Optical Clock Transitions in Yb+171 and Constraints on the Time Variation of Fundamental Constants, Phys. Rev. Lett. 113 (2014) 210801 [arXiv: 1407.0164 ] [INSPIRE].

[36] P. Touboul et al., MICROSCOPE Mission: First Results of a Space Test of the Equivalence Principle, Phys. Rev. Lett. 119 (2017) 231101 [arXiv:1712.01176] [InSPIRE].

[37] J. Bergé, P. Brax, G. Métris, M. Pernot-Borràs, P. Touboul and J.-P. Uzan, MICROSCOPE Mission: First Constraints on the Violation of the Weak Equivalence Principle by a Light Scalar Dilaton, Phys. Rev. Lett. 120 (2018) 141101 [arXiv:1712.00483] [INSPIRE]. 
[38] J.-P. Uzan, Varying Constants, Gravitation and Cosmology, Living Rev. Rel. 14 (2011) 2 [arXiv: 1009.5514] [INSPIRE].

[39] L. Heisenberg, M. Bartelmann, R. Brandenberger and A. Refregier, Dark Energy in the Swampland, arXiv: 1808.02877 [INSPIRE].

[40] T. Damour and J.F. Donoghue, Phenomenology of the Equivalence Principle with Light Scalars, Class. Quant. Grav. 27 (2010) 202001 [arXiv: 1007.2790] [INSPIRE].

[41] T. Damour and J.F. Donoghue, Equivalence Principle Violations and Couplings of a Light Dilaton, Phys. Rev. D 82 (2010) 084033 [arXiv: 1007.2792] [inSPIRE].

[42] M. Hohmann, L. Jarv, P. Kuusk and E. Randla, Post-Newtonian parameters $\gamma$ and $\beta$ of scalar-tensor gravity with a general potential, Phys. Rev. D 88 (2013) 084054 [Erratum ibid. D 89 (2014) 069901] [arXiv: 1309.0031] [INSPIRE].

[43] A. Schärer, R. Angélil, R. Bondarescu, P. Jetzer and A. Lundgren, Testing scalar-tensor theories and parametrized post-Newtonian parameters in Earth orbit, Phys. Rev. D 90 (2014) 123005 [arXiv: 1410.7914] [INSPIRE].

[44] L. Perivolaropoulos, PPN Parameter gamma and Solar System Constraints of Massive Brans-Dicke Theories, Phys. Rev. D 81 (2010) 047501 [arXiv:0911.3401] [InSPIRE].

[45] X.-M. Deng and Y. Xie, Solar System tests of a scalar-tensor gravity with a general potential: Insensitivity of light deflection and Cassini tracking, Phys. Rev. D 93 (2016) 044013 [INSPIRE].

[46] A. Arvanitaki, M. Baryakhtar and X. Huang, Discovering the QCD Axion with Black Holes and Gravitational Waves, Phys. Rev. D 91 (2015) 084011 [arXiv:1411.2263] [INSPIRE].

[47] G.L. Smith, C.D. Hoyle, J.H. Gundlach, E.G. Adelberger, B.R. Heckel and H.E. Swanson, Short range tests of the equivalence principle, Phys. Rev. D 61 (2000) 022001 [INSPIRE].

[48] E. Fischbach and C. Talmadge, Ten years of the fifth force, in Dark matter in cosmology, quantum measurements, experimental gravitation. Proceedings, 31st Rencontres de Moriond, 16th Moriond Workshop, Les Arcs, France, January 2-27, 1996, pp. 443-451 (1996) [hep-ph/9606249] [INSPIRE].

[49] A. Arvanitaki, J. Huang and K. Van Tilburg, Searching for dilaton dark matter with atomic clocks, Phys. Rev. D 91 (2015) 015015 [arXiv: 1405. 2925] [inSPIRE]. 